\title{
Propranolol inhibits cavernous vascular malformations by $\beta 1$ adrenergic receptor antagonism in animal models
}

\author{
Wenqing Li, ${ }^{1}$ Robert Shenkar, ${ }^{2}$ Mathew R. Detter, ${ }^{3}$ Thomas Moore, ${ }^{2}$ Christian Benavides, ${ }^{3}$ Rhonda Lightle, ${ }^{2}$ Romuald Girard, \\ Nicholas Hobson, ${ }^{2}$ Ying Cao, ${ }^{2}$ Yan Li, ${ }^{2,4}$ Erin Griffin, ${ }^{3}$ Carol Gallione, ${ }^{3}$ Joseph M. Zabramski, ${ }^{5}$ Mark H. Ginsberg, ${ }^{1}$ \\ Douglas A. Marchuk, ${ }^{3}$ and Issam A. Awad ${ }^{2}$ \\ 'Department of Medicine, UCSD, San Diego, California, USA. ${ }^{2}$ Department of Neurological Surgery, University of Chicago, Chicago, Illinois, USA. ${ }^{3}$ Department of Molecular Cenetics and Microbiology, \\ Duke University, Durham, North Carolina, USA. ${ }^{4}$ Bioinformatics Core, Center for Research Informatics, University of Chicago, Chicago, Illinois, USA. ${ }^{5}$ Department of Neurosurgery, Barrow Neurological \\ Institute, Phoenix, Arizona, USA
}

\begin{abstract}
Propranolol, a pleiotropic $\beta$-adrenergic blocker, has been anecdotally reported to reduce cerebral cavernous malformations (CCMs) in humans. However, propranolol has not been rigorously evaluated in animal models, nor has its mechanism of action in CCM been defined. We report that propranolol or its $\mathrm{S}(-)$ enantiomer dramatically reduced embryonic venous cavernomas in ccm 2 mosaic zebrafish, whereas R-(+)-propranolol, lacking $\beta$ antagonism, had no effect. Silencing of the $\beta 1$, but not $\beta 2$, adrenergic receptor mimicked the beneficial effects of propranolol in a zebrafish CCM model, as did the $\beta 1$-selective antagonist metoprolol. Thus, propranolol ameliorated cavernous malformations by $\beta 1$ adrenergic antagonism in zebrafish. Oral propranolol significantly reduced lesion burden in 2 chronic murine models of the exceptionally aggressive $P d c d 10 / C c m 3$ form of CCM. Propranolol or other $\beta 1$-selective antagonists may be beneficial in CCM disease.
\end{abstract}

\section{Introduction}

Cerebral cavernous malformations (CCMs) are vascular anomalies caused by mosaic inactivation of KRIT1 (also known as CCM1), CCM2, or PDCD10 (also known as CCM3) (1). We recently used CRISPR/Cas9 mutagenesis to mosaically inactivate zebrafish ccm2, resulting in multicavernous CCM-like lesions in the embryonic caudal venous plexus (CVP) caused by abortive intussusceptive angiogenesis (ref. 2 and Supplemental Figure 1; supplemental material available online with this article; https://doi.org/10.1172/ JCI144893DS1). In addition to its gross resemblance to CCM, the multicavernous CVP lesion shares a common pathogenesis with mammalian CCM through its initiation by mosaicism for CCM2 and its dependence on KLF2 transcription factors. Thus, the zebrafish CVP cavernoma serves as a facile in vivo model for rapid analysis of mechanisms of CCM formation.

Several case reports (3-7) and an ongoing randomized, controlled pilot trial, Treat_CCM (ClinicalTrials.gov NCT03589014) (8), have proposed treating CCMs with propranolol, a pleiotropic, nonselective $\beta$ adrenergic receptor blocker. It is unclear whether the anecdotally reported effects impact lesion growth or bleeding. Propranolol has been the frontline therapy for infantile hemangioma since 2015, when results from a randomized, controlled trial showed it to be effective (9), and it has been proposed for the treatment of other vascular abnormalities (10). The

Authorship note: WL and RS contributed equally to this work.

Conflict of interest: The authors have declared that no conflict of interest exists.

Copyright: ( 2021, American Society for Clinical Investigation.

Submitted: October 5, 2020; Accepted: December 1, 2020; Published: February 1, 2021.

Reference information: J Clin Invest. 2021;131(3):e144893.

https://doi.org/10.1172/JCl144893. mechanism of action of propranolol in vascular malformations is unclear; however, recent studies proposed that the effect of propranolol on infantile hemangiomas is ascribable to its capacity to inhibit SOX18 rather than to induce $\beta$ adrenergic antagonism (11). A potential effect of propranolol on CCM has not been investigated in animal models, and a mechanism of action has not been defined.

Zebrafish $\beta$-adrenergic receptor orthologs including adrb1 and adrb2a have been identified (12), and $\beta$ antagonists, such as propranolol and metoprolol, alter heart function in zebrafish, analogous to their effects in mammals $(13,14)$. Here, we investigated the effect of propranolol on embryonic zebrafish multicavernous malformation and on lesion burden and hemorrhage in 2 murine chronic CCM models. Using the zebrafish CVP model, we show that the beneficial effect could be ascribed to $\beta$-adrenergic receptor antagonism and that silencing of the $\beta 1$-adrenergic receptor had a similar beneficial effect on lesion formation. We conducted studies in murine models involving the loss of Ccm3/Pdcd10 because of this model's exceptional disease aggressiveness (15). The sensitized heterozygous $\left(\mathrm{Pdcd} 10^{+/-} \mathrm{Tr} p 53^{-/-}\right)$chronic disease model has already been used in several studies investigating the effect of other treatments on CCM disease, including Rock inhibition and B cell depletion $(16,17)$. More recently, the induced homozygous $\left(P d c d 1 O^{E C K O}\right)$ chronic disease model was used to determine the effect of Rock inhibition, tempol, and vitamin D on CCM lesions (18). We demonstrated that propranolol reduced lesion burden in all 3 animal models involving 2 species and different CCM genes. Effects were observed in both sexes. Thus, these preclinical studies provide support for clinical trials of propranolol or more $\beta 1$-selective antagonists in CCM. 


\section{Results and Discussion}

Racemic propranolol but not $R-(+)$-propranolol rescues cavernous vascular lesion development in ccm 2 CRISPR embryos. Propranolol is a nonselective $\beta$-antagonist and has a number of pleiotropic effects apart from $\beta$-adrenergic antagonism. For example, the beneficial effect of commercial racemic propranolol on infantile hemangioma has been ascribed to SOX18 inhibition by its $\mathrm{R}(+)$ enantiomer (13). To test the effect of propranolol on embryonic zebrafish cavernous malformations, we injected cas 9 mRNA and guide RNAs (gRNAs) targeting ccm2 into single-cell $\mathrm{Tg}$ (fli1:EGFP; gata1:DsRED) embryos. We added $100 \mu \mathrm{M}$ racemic, S-(-)-propranolol or R-(+)-propranolol 24 hours post fertilization (hpf) and imaged $\mathrm{GFP}^{+}$endothelial cells and DsRED ${ }^{+}$red blood cells 30, 36, and $48 \mathrm{hpf}$. In racemic propranolol-treated and S-(-)-propranolol-treated embryos, the CVP underwent a normal progression of sprouting angiogenesis and remodeling to form the dorsal and ventral veins interconnected by a venous plexus (Figure 1, A, B, E, $\mathrm{F}, \mathrm{I}$, and J). In sharp contrast, by $30 \mathrm{hpf}, 30 \%$ of the R-(+)-propranolol-treated (Figure 1C) and control (Figure 1D) embryos exhibited intussusceptive pillars (arrows) within the dorsal vein lumen. At $36 \mathrm{hpf}$, racemic propranolol-treated (Figure 1E) and S-(-)-propranolol-treated (Figure 1F) embryos formed a ventral vein, whereas the ventral vein did not form in R-(+)-propranolol-treated (Figure $1 \mathrm{G}$ ) or control (Figure $1 \mathrm{H}$ ) embryos, and intussusceptive pillars persisted within the dorsal vein. These pillars increased in length and number, restricting the flow of blood and resulting in dramatic dilation of the dorsal vein at $48 \mathrm{hpf}$ (Figure 1, K and L). Brightfield images illustrated the normal CVP development of racemic propranolol-treated (Figure $1 \mathrm{M}$ ) and S-(-)-propranolol-treated (Figure $1 \mathrm{~N}$ ) ccm 2 CRISPR embryos at $48 \mathrm{hpf}$, whereas $30 \%$ of R-(+)-propranolol-treated (Figure 1O) and control ccm 2 CRISPR embryos (Figure 1P) showed dramatic CVP dilation. As previously reported (2), approximately $10 \%$ of $c \mathrm{~cm} 2$ CRISPR embryos develop the $c c m 2$-null dilated heart phenotype, and propranolol did not affect this lethal phenotype (Figure 1, $\mathrm{Q}^{-\mathrm{T}}$ ). These results, quantified in Figure 1U, reveal that racemic propranolol prevented the formation of multicavernous malformations in $\mathrm{ccm} 2 \mathrm{CRISPR}$ embryos, whereas it had no effect on the dilated heart phenotype. Furthermore, it was the $\mathrm{S}$ enantiomer, which is primarily responsible for propranolol's $\beta$ adrenergic antagonism rather than the much less potent $\mathrm{R}$ enantiomer, that prevented cavernoma formation in the zebrafish caudal vein (Figure $1 \mathrm{~V}$ ).

Silencing the $\beta 1$ but not $\beta 2$ adrenergic receptor prevents embryonic cavernous vascular malformations. We hypothesized that propranolol inhibition of the dilated CVP in $c c m 2$ CRISPR embryos is due to $\beta 1$ adrenergic antagonism. To test this idea, we silenced the $\beta 1$ receptor using an adrb1 morpholino, resulting in virtually complete elimination of CVP dilation (Figure $2, \mathrm{~A}-\mathrm{C}$ ). In sharp contrast, silencing the $\beta 2$ a receptor, the predominant $\beta$ receptor in endothelium, had no such effect (Figure 2C). Furthermore, the $\beta 1$ selective antagonist metoprolol also prevented CVP dilation (Figure 2D). Thus, the ability of propranolol to inhibit multicavernous CVP lesions is ascribable to $\beta 1$ adrenergic receptor antagonism.

Propranolol decreases lesion burden in 2 chronic CCM murine models. These studies showed that propranolol could reduce cavernomas in an embryonic zebrafish model by $\beta 1$ antagonism. We then examined its effects on 2 chronic $C \mathrm{~cm} 3$ murine models of the most aggressive form of CCM in humans. We first demonstrated that the propranolol dose used in our murine study, $50 \mathrm{mg} / \mathrm{kg} / \mathrm{day}$, was sufficient to inhibit $\beta 1$ receptors in $P d c d 1 O^{E C K O}$ mice, as shown by a reduction in mean contractility (Figure $3 \mathrm{~A}$ ) and mean heart rate upon isoproterenol challenge (Figure $3 \mathrm{~B}$ ). With this same oral dose of $50 \mathrm{mg} / \mathrm{kg} /$ day propranolol treatment over 5 weeks, the mean lesion burden decreased $(P<0.0001)$ by $23 \%$ in the $P d c d 1 O^{E С K O}$ model and was halved $(P=0.014)$ in the $P d c d 10^{+/-} \operatorname{Tr} p 53^{-/-}$model (Figure $3 \mathrm{C}$ ). In the $P d c d 1 O^{E C K O}$ model, the majority of mice treated with propranolol had lesion burdens less than or equal to the mean placebo lesion burden ( $P=0.005$, Fisher's exact 2-tailed test). We note that lesions were mainly located in the hindbrain in the $P d c d 10^{E С К O ~ m o d e l ~ a s ~ a ~ r e s u l t ~ o f ~ g l o b a l ~ i n a c t i v a t i o n ~ o f ~} \mathrm{Ccm} 3$ at P6 (Figure 3D). In sharp contrast, in $\mathrm{Pdcd10^{+/- }} \mathrm{Trp} 53^{-/-}$mice, lesions were continuously being generated by random inactivation of $\mathrm{Ccm} 3$ due to global loss of Trp53, and the lesions were present in discrete masses in both the forebrain and hindbrain (Figure 3D). Despite these differences, propranolol significantly reduced lesions in both models. The wide variability in lesion burden in both models and its nonparametric distribution (few animals harboring a high lesion burden) are similar to the human disease, in which few patients manifest particularly large, aggressive lesions.

Out of 52 mice of the Pdcd1OECKO model (25 propranololtreated mice and 27 placebo controls, randomized for the assessment of lesion burden), 3 female mice underwent attrition before study completion (1 propranolol-treated mouse and 2 placebo-treated control mice were euthanized for morbidities, including 1 mouse with a large skin abscess). Propranolol treatment did not affect attrition compared with placebo controls in this model (Figure 3E). Similarly, out of 45 mice of the $\mathrm{Pdcd10^{+/- }}$ Trp $53^{-/-}$model (23 propranolol-treated mice and 22 placebo-treated controls), 9 male mice underwent attrition (4 propranolol-treated mice and 5 placebo-treated controls). These included 2 placebo-treated control mice found dead before study completion and the remaining 7 mice that were euthanized for morbidities, with 1 propranolol-treated mouse and 1 placebo-treated control mouse harboring tumors. Propranolol treatment did not affect attrition compared with placebo controls in this model (Figure 3F).

Propranolol therapy was commenced at weaning in the $\mathrm{Pdcd}_{10^{+/}} \mathrm{Trp}^{-/-}$model, when lesion burden is nil, and on day 21 in the $P d c d 10^{E C K O}$ model, when there is a very low lesion burden (18). Hence, neither experiment solely tested or established effects on a preexisting lesion. New models are being developed to test the effects of therapies on established lesions by creating a solitary CCM lesion via injection of adeno-associated virus vectors, encoding Cre recombinase, through a cranial window into the brains of Krit1 ${ }^{f / f l}$ adult mice. These studies are outside the scope of this Concise Communication.

Propranolol does not significantly affect hemorrhage in murine CCMs. Propranolol treatment did not affect acute bleeding in the brains of either the $P d c d 10^{E C K O}$ mice (Supplemental Figure 2, A and $\mathrm{B}$ ) or the $\mathrm{Pdcd10^{+/- }} \operatorname{Trp} 53^{-/-}$mice (Supplemental Figure 2, C and D). We observed no effect of propranolol on the number of induced homozygous chronic lesions in which inflammation $(P=$ $0.61)$ or nonheme iron deposition/chronic bleeding $(P=0.31)$ was detected (Fisher's exact test), both of which were uncommon in this model. In the $\mathrm{Pdcd10^{+/- }} \operatorname{Trp} 53^{-/-}$model, propranolol did not 
( \pm ) Propranolol
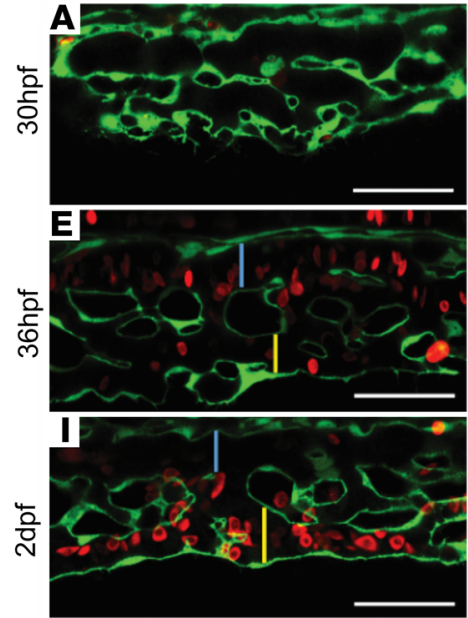

M
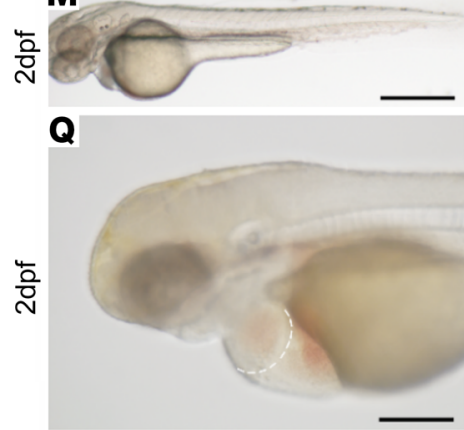

$\mathbf{U}$

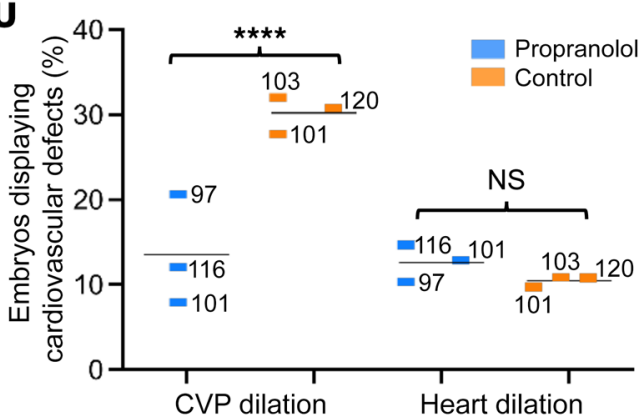

$\mathbf{N}$

R
S-(-)-propranolol
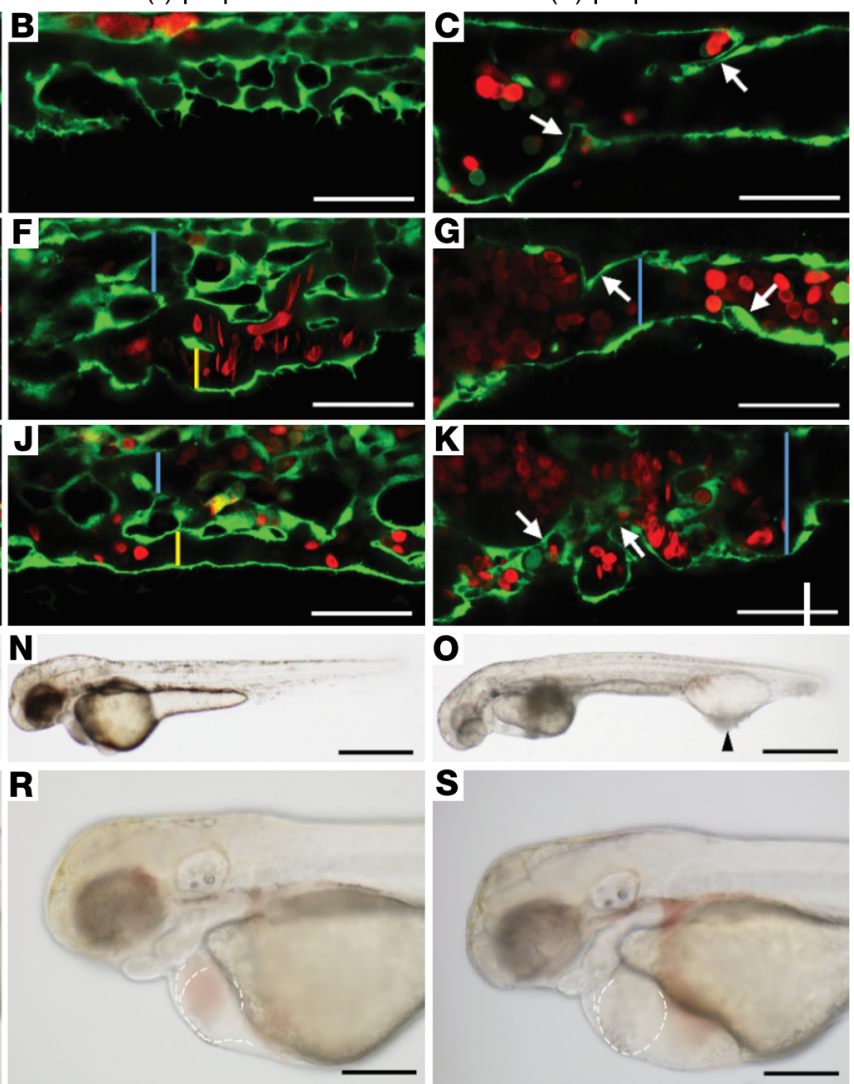

0
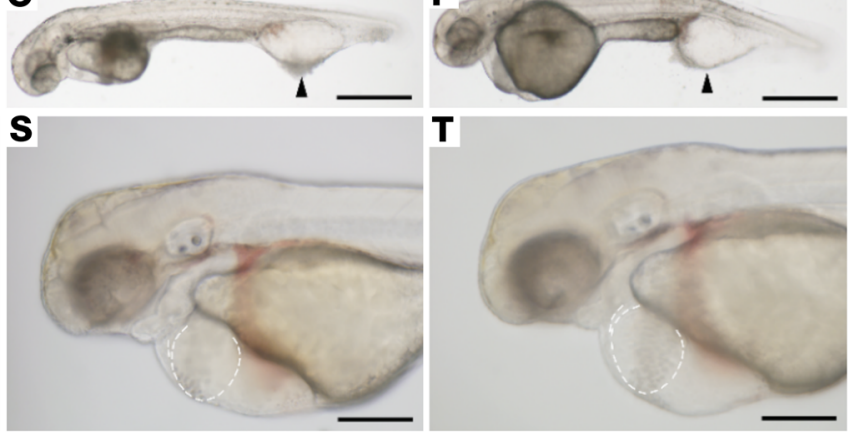

V

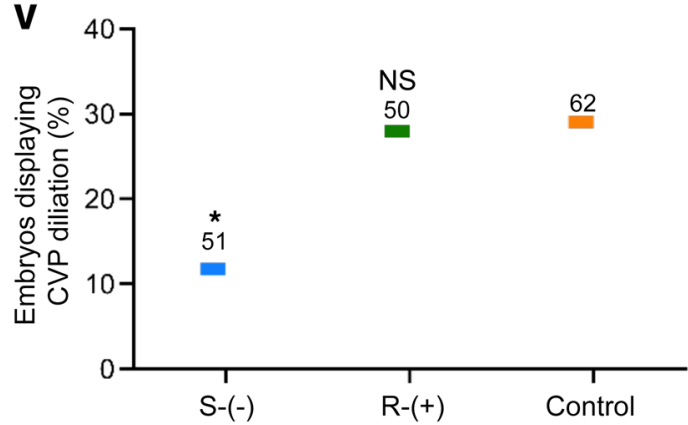

Figure 1. Propranolol and S-(-)-propranolol, but not R-(+)-propranolol, rescues embryonic CVP dilation in ccm2 CRISPR embryos. At the 1-cell stage, Tg(fli1:EGFP; gata1:DsRED) embryos, expressing EGFP in endothelial cells and DsRED in red blood cells, were injected with cas9 mRNA and gRNAs targeting ccm2. At $24 \mathrm{hpf}$, the ccm2 CRISPR embryos were treated with $100 \mu \mathrm{M}$ propranolol, S-(-)-propranolol, or R-(+)-propranolol. The CVP was scanned at 30 hpf (A-D), 36 hpf (E-H), and 2 days post fertilization (dpf) (I-L). Whereas R-(+)-propranolol-treated (C, G, and $\mathbf{K}$ ) and untreated CRISPR embryos (D, H, and $\mathbf{L}$ ) displayed abnormal intussusceptions within the dorsal vein and lack of a ventral vein, racemic propranolol-treated (A, E, and I) and S-(-)-propranolol-treated (B, F, and J) CRISPR embryos formed the primitive venous plexus by $30 \mathrm{hpf}(\mathbf{A}$ and $\mathbf{B})$, a normal functional ventral vein by $36 \mathrm{hpf}(\mathbf{E}$ and $\mathbf{F})$, and a mature caudal venous plexus by $2 \mathrm{dpf}$ (I and $\mathbf{J})$. Arrows indicate intussusceptions. Blue and yellow bars indicate the dorsal and ventral veins, respectively. The embryos shown in A-T are in the sagittal plane. Bright-field images revealed normal CVP development of racemic propranolol-treated (M) and S-(-)-propranolol-treated CRISPR embryos (N), whereas approximately 30\% of R-(+)-propranolol-treated (0) and untreated (control) ccm2 CRISPR embryos (P) had dramatic CVP dilation. Approximately $10 \%$ of embryos in all 4 groups showed heart dilation (Q-T). Scale bars: $50 \mu \mathrm{m}(\mathbf{A}-\mathbf{L}), 500 \mu \mathrm{m}(\mathbf{M}-\mathbf{P})$, and $200 \mu \mathrm{m}(\mathbf{Q}-\mathbf{T})$. Quantitation of ccm2 CRISPR embryos with cardiovascular defects (U) revealed that, compared with the untreated controls, significantly fewer propranolol-treated embryos harbored CVP dilation ( $\left.{ }^{* * *} P<0.0001\right)$, whereas a similar proportion exhibited heart dilation $(P=0.11)$. Quantitation of ccm2 CRISPR embryos with CVP dilation (V) showed that the S-(-)-propranolol enantiomer rescued CVP dilation $\left({ }^{*} P=0.036\right)$, whereas the R-(+)-propranlol enantiomer failed to do so $(P=1.0)$ compared with untreated control embryos. A 2-tailed Fisher exact test was used for comparisons. Each replicate (colored bars) is presented with the total number. Black bars indicate the mean in $\mathbf{U}$.

affect chronic hemorrhage from CCM lesions as measured by iron deposition (Supplemental Figure 2E). These observations make it unlikely that propranolol restricts the growth of CCM lesions by preventing hemorrhage.
Propranolol decreases multicavernous lesions, but not single cav-

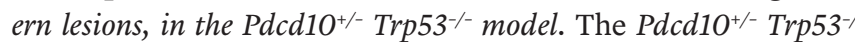
model allows the exploration of lesion development versus maturation $(14,16)$. Propranolol did not affect $(P=0.178)$ the burden 

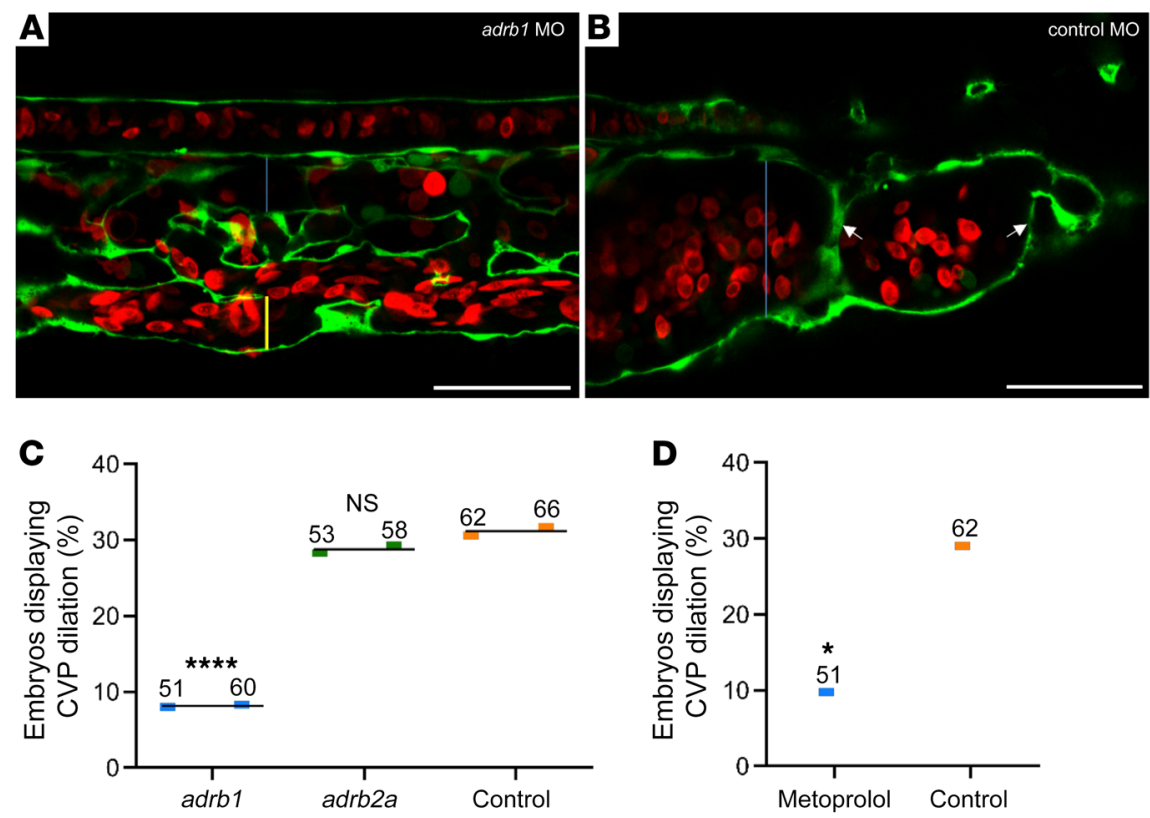

Figure 2. Loss of $\boldsymbol{\beta} 1$ adrenergic receptor function inhibits CVP dilation in ccm2 CRISPR embryos. (A) One-cell-stage embryos were coinjected with ccm2 CRISPR and an adrb1 morpholino (MO) (A and C) to silence the $\beta 1$ receptor or with a control morpholino (B and C) and imaged at 48 hpf. (A) $\beta 1$ Adrenergic receptor-silenced embryos formed a normal CVP, whereas control morpholino embryos (B) showed dramatic dilation. The blue bar indicates the lumen of the dorsal vein, and the yellow bar indicates the ventral vein. Scale bars: $50 \mu \mathrm{m}$. (C) Quantification of the embryos in replicate experiments showed that CVP dilation in ccm2 CRISPR embryos was reversed by silencing of the $\beta 1$ adrenergic receptor with an adrb1 $1^{-/-}$morpholino $\left({ }^{* * *} P<0.0001\right)$, but not by silencing of the $\beta 2$ a adrenergic receptor with an adrb2a-/- morpholino $(P=0.89)$. (D) Specificity of $\beta$ adrenergic antagonists. The $\beta 1$-specific antagonist metoprolol ( $400 \mu \mathrm{M})$ was added $24 \mathrm{hpf}$, and embryos were imaged $48 \mathrm{hpf}$. Metoprolol reversed CVP dilation ( $\left.{ }^{*} P=0.018\right)$ in comparison with untreated control embryos. The fraction of defective embryos for each replicate experiment is indicated by colored bars (C and $\mathbf{D}$ ). The mean of the values from replicate experiments is indicated by thin black lines (C). The numbers above the colored bars indicate the sample size for each replicate experiment in each group (C and D). A 2-tailed Fisher's exact test was used for comparisons.

of stage 1 single-cavern lesions (Supplemental Figure 3A). In contrast, the mean \pm SEM burden of stage 2 mature multicavernous lesions was halved $(P=0.004)$ with propranolol treatment (Supplemental Figure 3A). This effect of propranolol on lesion maturation, rather than lesion genesis, contrasts sharply with the effect of fasudil, which reduces both stage 1 and stage 2 lesions (19).

Stage 1 lesions are known to be composed entirely of Ccm3-null endothelial cells, whereas stage 2 lesions are mosaic, containing both wild-type and Ccm3-null cells (20, 21). We recently proposed that blood flow might stimulate the formation of stage 2 mosaic multicavernous lesions and disfavor the formation of global-knockout stage 1 vascular malformations in zebrafish (2). The present results show that propranolol, possibly by changing blood flow, had a similar selective effect on murine stage 2 lesions. The $P d c d 10^{E C K O}$ model allowed the examination of a therapeutic effect in both sexes and demonstrated a similar benefit in male and female mice (Supplemental Figure 3B).

Therapeutic implications. Zebrafish experiments allowed the testing of mechanistic hypotheses in a vascular lesion with multicavernous histology similar to that of human CCM, resulting from $\mathrm{Ccm} 2$ gene loss and dependent on KLF2 transcription factors. We conducted mouse experiments in more aggressive CCM3 models and observed robust brain lesion development that allowed preclinical testing of oral propranolol therapy in different models and genotypes. The effect of propranolol in murine models was quite modest, primarily decreasing the prevalence of mice with high lesion burdens. The primary outcome (lesion volume/brain vol- ume) was in a range similar to the reported effects (Supplemental Figure $3 \mathrm{C}$ ) of the Rock inhibitor fasudil, the HMG CoA reductase inhibitor atorvastatin (16), or B cell depletion (17) in the same $P d c d 10^{+/-} \operatorname{Trp} 53^{---}$model. However, fasudil, atorvastatin, and B cell depletion all inhibited chronic hemorrhage from the CCM lesions in the same model in the aforementioned studies, in contrast to the failure of propranolol to reduce lesional bleeding in the present study. Conversely, in the $P d c d 10^{E C K O}$ model, fasudil and tempol (18) failed to significantly reduce lesion burden, while propranolol was effective. Both lesion growth and hemorrhage are important clinically. Different drugs appear to have varying effects on CCM lesion development and hemorrhage, hence, these features need to be specifically examined in animal models and the different endpoints carefully tracked in clinical studies.

We examined oral propranolol at a dose sufficient to inhibit $\beta 1$ receptors in $P d c d 10^{\text {EСКO }}$ mice. Depending on the signal effects in the ongoing pilot propranolol trial (8) and subjects' tolerance to side effects, we propose that propranolol dose escalation might be considered in future human clinical trials at doses that effect physiologic $\beta 1$ receptor inhibition. $\beta 1$ Adrenergic antagonists ameliorate some of the dose-limiting side effects of propranolol such as bronchospasm. Thus, our finding that the beneficial effects of propranolol may be ascribed specifically to $\beta 1$ adrenergic antagonism suggests that the use of such agents alone, or in combination with other drugs such as Rho kinase inhibitors $(18,22,23)$, merits investigation and may find a place in CCM therapy. 

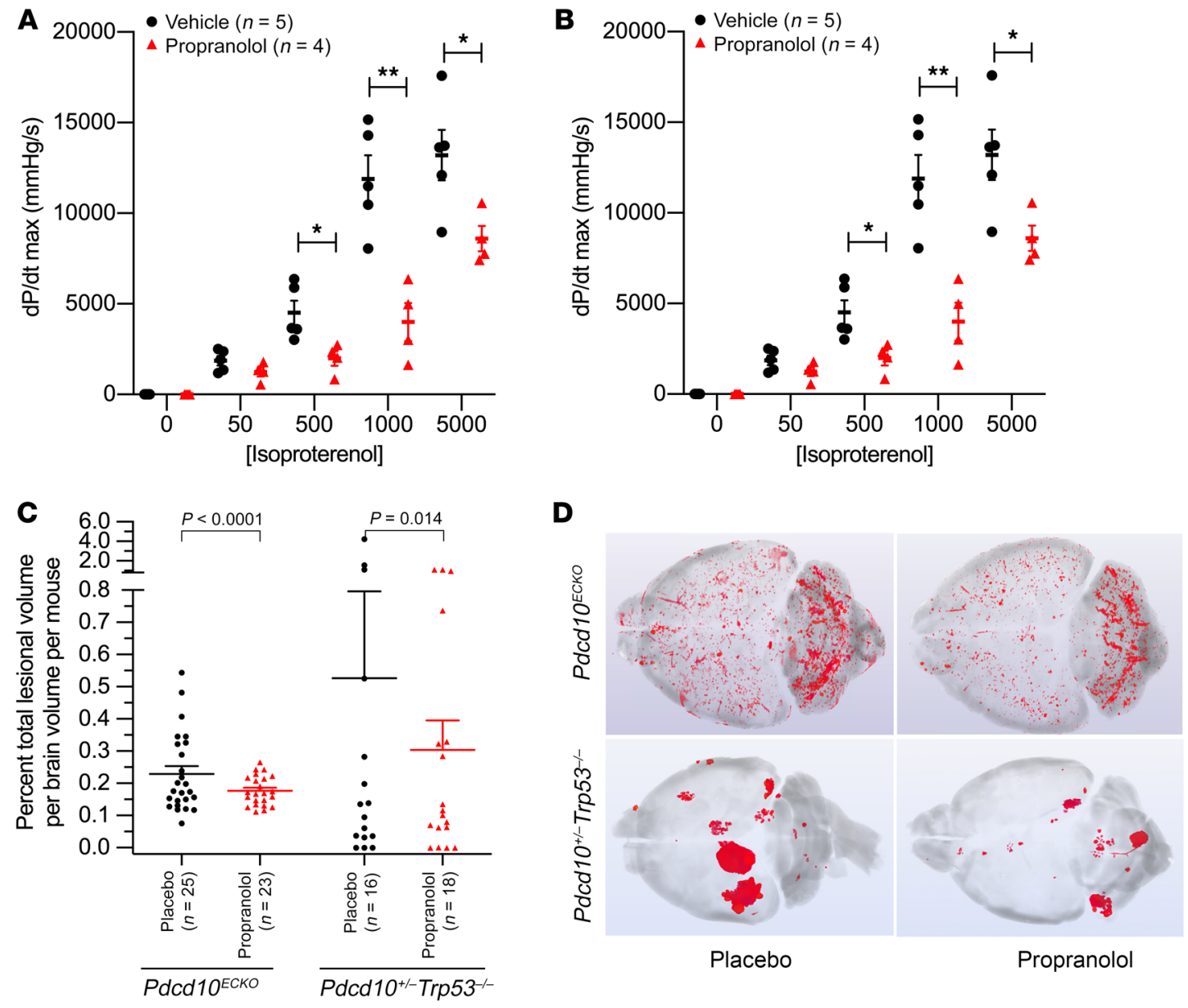

Placebo

Propranolol
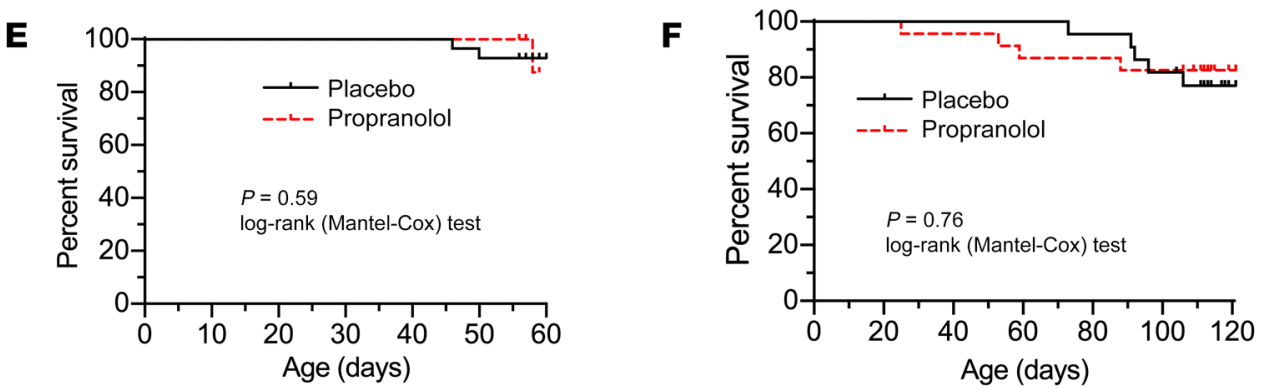

Figure 3. Effect of propranolol in murine models of CCM disease. $P d c d 10^{E C K O}$ mice, treated with placebo $(n=5)$ or propranolol $(n=4)$, were challenged with increasing doses of isoproterenol (50-5000 pg) to determine whether the $50 \mathrm{mg} / \mathrm{kg} /$ day propranolol dose was sufficient to antagonize $\beta$-adrenergic receptors. Propranolol-treated mice showed a significant rightward shift in the dose response curve in (A) contractility (dP/dt max) and (B) heart rate (bpm) with increasing concentrations of isoproterenol. Data are expressed as the mean $\pm \mathrm{SEM}$. ${ }^{*} P<0.05$ and ${ }^{* *} P<0.01$, by 2 -way repeated-measures ANOVA with a post hoc independent samples $t$ test. (C) Effect of propranolol on lesion burden in 2 Pdcd10 models. In the Pdcd10 ECKO model, propranolol (50 mg/kg/day) for 35 days (dose started on P21) significantly decreased lesion burden compared with placebo controls $(P<0.0001)$. One placebo-treated mouse was excluded as an outlier. In the $P d c d 10^{+-} \operatorname{Trp} 53^{--}$model, propranolol $(50 \mathrm{mg} / \mathrm{kg} /$ day) for 90 days (dose started on P21) significantly reduced lesion burden compared with placebo controls $(P=0.014)$. One placebo-treated mouse and 1 propranolol-treated mouse were excluded as outliers. Since the data were not normally distributed, the 2-sample Conover test was used. The mean (longer bars) and SEM (shorter lines above the mean bars) are indicated for each group. In both the $P d c d 10^{E C K O}$ and $P d c d 10^{+/-} \operatorname{Trp}_{53} 3^{-/-}$models, the lesion burden was greater in the placebo-treated controls than in the propranolol-treated mice. (D) Representative micro-CT images of the brains of mice with a high lesion burden in the untreated groups and average mouse brains in the treated groups, showing the effect of propranolol primarily on animals with a high lesion burden. Propranolol did not affect survival in either the $(\mathbf{E}) P d c d 10^{E C K O}$ model $(P=0.59)$ or the (F) $P d c d 10^{+/-} \operatorname{Trp}^{2} 3^{-/-}$model $(P=0.76)$ compared with placebo controls. The log-rank (Mantel-Cox) test was used to determine significance.

\section{Methods}

Detailed methods are provided in the Supplemental Methods.

Study approval. All experiments were approved by the IACUCs of UCSD and Duke University.

\section{Author contributions}

IAA, MHG, DAM, and JMZ conceptualized the study. WL and MHG conducted and analyzed the zebrafish experiments. MRD, $\mathrm{CB}, \mathrm{EG}, \mathrm{CG}$, and DAM conducted the mouse experiments. RS, 
TM, RG, and RL analyzed the mouse data. TM, RL, RG, and NH conducted the micro-CT studies. RS, YC, and YL performed the statistical analysis. WL and RS drafted the manuscript. MHG, DAM, and IAA oversaw edits and revisions.

\section{Acknowledgments}

This work was supported by grants from the NIH (2P01 NS092521, to DAM, IAA, and MHG; F30 HL140871, to MRD; R01 HL056687, HL075443, and R01 HL075443, to Howard A. Rockman, and T32 GM007171, to MRD); the American Heart Association
(18PRE34060061, to MRD); the Fondation Leducq (17 CVD 03, to DAM); and Be Brave for Life (to WL, JMZ, DAM, and IAA). The authors would like to thank Wang Min for providing the transgenic mice used in this work. We thank the Duke Cardiovascular Research Center Small Animal Physiology Core for performing the in vivo mouse hemodynamic experiments.

Address correspondence to: Issam A. Awad, Department of Neurological Surgery, University of Chicago, 5841 S. Maryland Ave., MC 3026, Chicago, Illinois 60637, USA. Phone: 773.702.2123; Email: iawad@uchicago.edu.
1. Leblanc GG, et al. Biology of vascular malformations of the brain. Stroke. 2009;40(12):e694-e702.

2. Li W, et al. Abortive intussusceptive angiogenesis causes multi-cavernous vascular malformations [preprint]. https:// doi:10.1101/2020.08.14.251744. Posted on bioRxiv. August 14, 2020.

3. Ghosh PS, Ghosh D. Infantile intraspinal and extensive cutaneous hemangiomas: excellent response to propranolol. Neurology. 2011;76(20):1771.

4. Goldberg J, et al. Bleeding risk of cerebral cavernous malformations in patients on $\beta$-blocker medication: a cohort study [Published online June 15, 2018]. J Neurosurg. https://doi. org/10.3171/2017.12.JNS172404.

5. Miquel J, et al. Successful treatment of multifocal intracerebral and spinal hemangiomas with propranolol. J Am Acad Dermatol. 2014;70(4):e83-e84.

6. Moschovi M, et al. Propranolol treatment for a giant infantile brain cavernoma. J Child Neurol. 2010;25(5):653-655.

7. Reinhard M, et al. Propranolol stops progressive multiple cerebral cavernoma in an adult patient. J Neurol Sci. 2016;367:15-17.

8. Lanfranconi S, et al. Propranolol for familial cerebral cavernous malformation (Treat_CCM): study protocol for a randomized controlled pilot trial. Trials. 2020;21(1):401.

9. Leaute-Labreze C, et al. A randomized, controlled trial of oral propranolol in infantile hemangioma. N Engl JMed. 2015;372(8):735-746.

10. Trenor CC 3rd. Medical management of vascular anomalies. Semin Cutan Med Surg. 2016;35(3):177-181.

11. Overman J, et al. R-propranolol is a small molecule inhibitor of the SOX18 transcription factor in a rare vascular syndrome and hemangioma. eLife. 2019;8:e43026.

12. Wang Z, et al. Zebrafish beta-adrenergic receptor mRNA expression and control of pigmentation. Gene. 2009;446(1):18-27.

13. Finn J, et al. Effects of propranolol on heart rate and development in Japanese medaka (Oryzias latipes) and zebrafish (Danio rerio). Aquat Toxicol. 2012;122-123:214-221.

14. Hsu JJ, et al. Contractile and hemodynamic forces coordinate notch1b-mediated outflow tract valve formation. JCI Insight. 2019;5(10):e124460.

15. Shenkar R, et al. Exceptional aggressiveness of cerebral cavernous malformation disease associated with PDCD10 mutations. Genet Med. 2015;17(3):188-196.

16. Shenkar R, et al. Rho kinase inhibition blunts lesion development and hemorrhage in murine models of aggressive Pdcd10/Ccm 3 disease. Stroke. 2019;50(3):738-744.

17. Shi C, et al. B-cell depletion reduces the maturation of cerebral cavernous malformations in murine models. J Neuroimmune Pharmacol. 2016;11(2):369-377.

18. Detter MR, et al. Novel murine models of cerebral cavernous malformations. Angiogenesis. 2020;23(4):651-666.

19. McDonald DA, et al. Fasudil decreases lesion burden in a murine model of cerebral cavernous malformation disease. Stroke. 2012;43(2):571-574.

20. Malinverno M, et al. Endothelial cell clonal expansion in the development of cerebral cavernous malformations. Nat Commun. 2019;10(1):2761.

21. Detter MR, et al. Cerebral cavernous malformations develop through clonal expansion of mutant endothelial cells. Circ Res. 2018;123(10):1143-1151.

22. Stockton RA, et al. Cerebral cavernous malformations proteins inhibit Rho kinase to stabilize vascular integrity. J Exp Med. 2010;207(4):881-896.

23. McKerracher L, et al. A brain-targeted orally available ROCK2 inhibitor benefits mild and aggressive cavernous angioma disease. Transl Stroke Res. 2020;11(3):365-376. 